\title{
Structure Modeling and Mechanical Analyses of Meniscal Implants based on Triply Periodic Minimal Surfaces
}

\author{
Shi Jianping ${ }^{1 *}$, Zhu Liya ${ }^{1}$, Yang Jiquan ${ }^{1}$ \\ ${ }^{1}$ Nanjing Normal University \\ No. 2 Xuelin Road, Nanjing 210046, China \\ jpshi@njnu.edu.cn; 61193@njnu.edu.cn; 63047@njnu.edu.cn
}

\section{Extended Abstract}

As an important part of the knee joint, the meniscus plays a role in transmitting load, absorbing oscillation, and improving joint stability [1]. However, joint disease, degeneration, trauma and other causes may cause damage to the meniscus. Meniscus transplantation can solve diseases such as osteoarthritis caused by meniscus loss, but there are some problems such as limited stent replacement, immune response, and structural mismatch [2,3]. There is currently a problem of mismatch in mechanical properties between commercial meniscus implants and natural meniscus, which is not conducive to long-term implantation [4]. Therefore, a porous polycarbonate-polyurethane meniscus implant based on a very small threeperiod surface is proposed.

First, a 3D model of the knee joint was established based on the CT scan results of the knee joint [5]. The porous element is constructed by Primitive minimal surface. After Boolean operation with the outer meniscus structure, a series of implant structures with different pore sizes or porosity are obtained by adjusting the surface construction parameters. Then, finite element simulation was performed to compare the mechanical changes of articular cartilage and bilateral meniscus in the knee joint of the natural meniscus and the designed porous meniscus.

The results show that the use of a porous meniscal implant can effectively reduce the compressive stress and shear stress concentration on the femoral cartilage and the tibial cartilage. At the same time, changes in the structural parameters of the porous implant affect the stress of the articular cartilage. In addition to having good mechanical properties, the structure can also be rapidly formed by three-dimensional printing technology, which provides a new idea for clinical application.

\section{References}

[1] A. L. Mcnulty and F. Guilak, "Mechanobiology of the meniscus," J. Biomech., vol. 48, no. 8, pp.1469-1478, 2015.

[2] I. Mcdermott, "Meniscal tears, repairs and replacement: their relevance to osteoarthritis of the knee," Brit J Sport Med., vol. 45, no. 4, pp. 292-299, 2011.

[3] J. Hasan, J. Fisher and E. Ingham, "Current strategies in meniscal regeneration," J. Biomed Mater Res B., vol. 102, no. 3, pp.619-634, 2014.

[4] Vrancken. A. C. T. and Pieter Buma, "Synthetic meniscus replacement: a review," Int Orthop., vol. 37, no. 2, pp.291299, 2013.

[5] Yoo, Dong J, "Porous scaffold design using the distance field and triply periodic minimal surface models," Biomaterials, vol. 32, no. 31, pp. 7741-7754, 2011. 